Revisión Breve/Brief Review

Efecto del tabaco en células madre de la cavidad bucal. Una revisión breve.

\title{
Effect of tobacco on oral cavity stem cells. A brief review.
}

\author{
Cifuentes-Suazo Gloria ${ }^{1}$, Stange-Dempster Felipe ${ }^{1}$, Cartes-Velásquez Ricardo ${ }^{2}$ \\ ${ }^{1}$ Facultad de Odontología, Universidad Andrés Bello, Concepción, Chile. \\ ${ }^{2}$ Fundación Kimntrum, Concepción, Chile.
}

\author{
Correspondencia/Corresponding \\ Dr. Ricardo Cartes-Velásquez: \\ Beltrán Mathieu 7, Concepción, Chile. \\ Correo electrónico / E-mail: cartesvelasquez@gmail.com
}

\section{ORCID}

Gloria Cifuentes-Suazo: https://orcid.org/0000-0002-6497-3480

Felipe Stange-Dempster: https://orcid.org/0000-0003-3697-1899

Ricardo Cartes-Velásquez: https://orcid.org/0000-0001-5831-7324

Received: 20 October 2020; Accepted: 11 May 2021; Published: 4 August 2021

\begin{abstract}
In recent years, mesenchymal stem cells (MSCs) have become the cornerstone of tissue engineering and regenerative medicine. MSCs can be easily obtained from adult tissues, it is in this area that obtaining and using oral MSCs have become a good source of experimentation. It is not unknown that MSCs have great regenerative power, high proliferative and differentiation capacity and high osteogenic potential. However, they can be affected by multiple external factors. The objective of this article is to describe the effect of tobacco on oral MSCs. Cigarettes and its components are a risk factor for various diseases at the level of the oral cavity, they have many adverse effects on the cellular biology of the mouth, therefore, it is not strange to think about the effect that these components can have on MSCs. of oral origin. The latest studies have shown that nicotine and condensed cigarette smoke have negative biological effects on MSCs, particularly oral ones. Despite the existing literature, it is not entirely clear what are the mechanisms involved in the negative effects of tobacco on oral MSCs and how they could be reversed.
\end{abstract}

Keywords: stem cells, tobacco, nicotine, oral cavity.

\section{Resumen}

En los últimos años, las células madres mesenquimales (CMM) se han convertido en la piedra angular de la ingeniería tisular y medicina regenerativa. Las CMM se pueden obtener fácilmente de los tejidos adultos, es en este ámbito que la obtención y utilización de las CMM orales, se han convertido en una buena fuente de experimentación. No es desconocido que las CMM tienen gran poder regenerativo, alta capacidad proliferativa, de diferenciación y alto potencial osteogénico. Sin embargo, pueden ser afectados por múltiples factores externos. El objetivo de este artículo es describir el efecto del tabaco sobre las CMM orales. El cigarrillo y sus componentes son un factor de riesgo para varias enfermedades a nivel de cavidad oral, tienen muchos efectos adversos en la biología celular de la boca, por eso, no se hace extraño pensar en la afectación que estos componentes pueden tener en las CMM de origen oral. Los últimos estudios realizados demuestran que la nicotina y el humo condensado del cigarrillo tiene efectos biológicos negativos sobre las CMM, en particular de las orales. A pesar de la literatura 
existente, no está del todo claro cuáles son los mecanismos involucrados en los efectos negativos del tabaco sobre las CMM orales y cómo podrían ser revertidos.

Palabras clave: células madre, tabaco, nicotina, cavidad oral.

\section{Introducción}

Existen diversos tipos de células madres $(\mathrm{CM})$, se ha descrito que todas poseen capacidad de autorrenovación y capacidad de diferenciación en los tejidos adultos. Las CM mesenquimales (CMM) han tomado un rol importante en la ingeniería tisular y medicina regenerativa, debido a su capacidad de sintetizar citocinas y factores de crecimiento que aumentan la dinámica celular local, lo cual les dan un alto potencial de regeneración y reparación de tejidos ${ }^{1,2}$. Además de su efecto inmunomodulador, las CMM liberan factores proangiogénicos y quimiotácticos, los cuales juegan un papel esencial en la cicatrización de heridas, remodelación ósea y regeneración tisular ${ }^{2}$.

Además de tener la capacidad de autorenovación, las CMM tienen la capacidad de migración celular. Las CMM migran hacia los sitios de lesión promoviendo la cicatrización de heridas, esto se logra mediante la liberación de citoquinas, factores de crecimiento y diferenciación directa, a este tipo de migración guiada se denomina "búsqueda de células madres" y es exclusiva de las CMM, lo que permite que las poblaciones celulares trasplantadas o residentes logren un suministro dirigido a las áreas enfermas $^{3}$. Las CMM tienen la capacidad de formar una gran variedad de tipos celulares ${ }^{3}$. De igual manera el potencial de diferenciación osteogénica ha sido ampliamente descrito en la literatura, llegando a ser considerado habitualmente en enfermedades metabólicas óseas y fijación de estructuras ${ }^{4-6}$

En la cavidad oral se describen ocho poblaciones de células madres mesenquimales (CMM): CM de la pulpa dental postnatal, $\mathrm{CM}$ de dientes deciduos exfoliados humanos, $\mathrm{CM}$ del ligamento periodontal, $\mathrm{CM}$ progenitoras del folículo dental, CM alveolares derivadas del hueso, CM de papila apical, CM progenitoras del germen dentario y CM gingivales. En la literatura queda evidenciado que todas las CMM de origen oral tienen la capacidad de diferenciarse en múltiples linajes, regenerar, inmunomodular y reparar muchos tipos de tejidos ${ }^{7-9}$.

El humo del cigarrillo contiene más de 7000 compuestos químicos, de la cuales aproximadamente 250 de ellos se conocen ${ }^{10}$, los componentes principales corresponden a la fase particulada, conocida como humo condensado, la cual contiene nicotina, fenol, hidrocarburos, nitrosaminas, metales pesados y diversos agentes carcinogénicos ${ }^{11}$. La exposición al humo del tabaco puede ocurrir de diferentes maneras, lo cual también influye directamente en la absorción de los componentes. La inhalación de primera mano o la convencional, es decir, aquella a la que se exponen los fumadores activos al contacto con el extremo filtrado, es la forma predominante de toxicidad directa. La segunda mano o corriente secundaria es aquella producida por el humo del extremo encendido del cigarrillo ${ }^{12}$.

La nicotina, el principal componente del cigarrillo, induce estrés oxidativo y activa respuestas inflamatorias e inmune de manera patológica ${ }^{13}$. Observando estos fenómenos, grupos de estudiosos comenzaron su investigación en relación al efecto que puede tener sobre las células madres. El objetivo de este artículo es describir el efecto del tabaco sobre las CMM orales.

\section{Efecto del tabaco sobre las células madres}

Durante muchos años se han estudiado diferentes factores que influyen de manera directa en la capacidad y potencial regenerativo de las CM, uno de estos factores es el humo del tabaco, principalmente para nicotina.

La explicación para todas las interacciones que la nicotina tiene en las células madres, es debido a que esta puede interactuar a través de receptores nicotínicos, conocidos como receptores nicotínicos de acetilcolina (nAChR) de los cuales se han identificado 16: a1-7, a9, a10, b1-4, g, d y e ${ }^{14}$. Estudios revelaron que las CMM solo poseían proteínas para las subunidades a7, b2 y b4 ${ }^{15}$. Sin embargo, posteriormente se reveló que el único receptor nicotínico funcional presente en las CMM es el receptor $a 7^{16}$. Estos receptores actúan como una puerta específica de calcio, al momento de tener nicotina en el sistema, la cual es antagonista de este receptor, aumentan los niveles de calcio intracelular, además la nicotina induce a un aumento en la fosforilación de las proteínas quinasas, las cuales desarrollan un 
papel fundamental en la proliferación y diferenciación de las $\mathrm{CM}^{17}$. Más aun, la nicotina desempeña un papel fundamental en la regulación de procesos celulares como la proliferación, migración y diferenciación, lo cual afecta el desarrollo de las células madres y tiene un efecto biológico negativo sobre estas ${ }^{18}$. Datos recientes han revelado que el humo del cigarrillo conduce a una disminución del potencial regenerativo, además de disminuir la proliferación, migración y potencial de diferenciación de las $\mathrm{CMM}^{19}$.

Por ejemplo, CMM derivadas de adipocitos expuestos a distintas concentraciones de nicotina no mostraron viabilidad, sin embargo, a una exposición de $1 \%$ no mostraron cambios significativos de viabilidad, lo cual demuestra la dosis dependencia ${ }^{20}$. De igual manera se observa una disminución en la migración celular de las CMM que es dosis dependiente, en concentraciones de $1 \%$ de humo de tabaco no existe mayor variabilidad en la migración, pero sí se ve gravemente afectado en concentraciones mayores al $5 \%{ }^{20}$. Por otro lado, la nicotina lleva a un aumento en la proporción de células que entran a fase G0/G1 ${ }^{21}$. G0 es considerada la etapa de reposo en el ciclo celular y que las células se encuentren en esta fase, puede explicar de alguna manera la disminución en la proliferación inducida por la nicotina ${ }^{22}$. Referente a la capacidad osteogénica, se ha visto que los fumadores tienen mayor probabilidad de experimentar osteoporosis y retraso en los tiempos de curación posterior a una fractura ${ }^{23-25}$. Estas demoras se deben en gran parte a la ineficaz diferenciación osteogénicas de las $\mathrm{CMM}^{20}$.

\section{Efecto del tabaco sobre las células madres orales}

Los componentes del humo del tabaco son perjudiciales para cualquier grupo celular, lo cual ha sido evidenciado ampliamente, además el cigarrillo es uno de los factores de riesgo de lesiones orales de gran importancia tales como: periodontitis, cáncer oral, lesiones ulcerativas, etc. ${ }^{26}$

La internalización de las toxinas se realiza principalmente a través del sistema respiratorio, pero para compuestos específicos como la nicotina, la absorción en tejidos depende mayoritariamente del $\mathrm{pH}$ de este, la nicotina es una base débil, por ende, su absorción se ve facilitada en medios ligeramente básicos. El sitio inicial de exposición al humo del tabaco es la cavidad oral, la cual se considera un ambiente más bien ácido, lo cual nos haría pensar que la absorción de la nicotina no es eficiente, sin embargo, informes actuales indican que el humo del cigarrillo es más alcalino, mejorando así la absorción a nivel de cavidad oral $^{27}$.

Estudios demuestran que la nicotina queda en la saliva, ya que este componente queda atrapado de forma ionizada manteniendo niveles elevados de exposición en la boca, se midió la concentración de nicotina en la saliva y arrojó que es 87 veces mayor que en el plasma sanguíneo ${ }^{28}$.

Es sabido que el tabaco regula la aparición y progresión de la enfermedad periodontal, la nicotina destruye o retrasa la regeneración de los tejidos periodontales, principalmente inhibiendo la proliferación de las células del ligamento periodontal a través de diversos mecanismos.

La nicotina aumenta la expresión de miR-30a el cual regula la expresión de CE2, lo cual conlleva a una inhibición de la proliferación celular y detención del ciclo celular. Se puede decir lo siguiente, el miR30a regulado por nicotina detiene el ciclo celular en la fase G1 al atacar directamente a CE2 en las $\mathrm{CLPH}^{29}$. Esto ha sido refrendado en otros estudios, donde se demostró que la nicotina tiene efectos inhibidores en la viabilidad celular y proliferación de las células del ligamento periodontal, siendo este efecto dependiente de la concentración y del tiempo ${ }^{30}$. Más aun, la nicotina inhibe el aumento del potencial osteogénico de las células del ligamento periodontal inducida por tensión cíclica, esto al unirse a un receptor de acetilcolina, el a7 nicotínico ${ }^{31}$

La evidencia indica que la nicotina es capaz de producir citocinas inflamatorias y especies reactivas de oxígeno en los cementoblastos ${ }^{32}$, inhibición de síntesis de proteínas en los fibroblastos del ligamento periodontal ${ }^{33}$, aumento de la actividad de la fosfatasa alcalina y estimulación de la síntesis de proteínas y colágeno en las células óseas ${ }^{34,35}$. En base a estos resultados el estudiar sus efectos en las células madres se vuelve fundamental. Uno de los mecanismos de la nicotina la inducción de actividad autofágica en las CM periodontales ${ }^{36}$.

\section{Efecto del tabaco sobre las capacidades regenerativas de las células madres orales}

Uno de los pocos estudios clínicos, aisló CMM provenientes de la pulpa dental de terceros molares impactados sanos, para esto se recolectaron muestras de 10 pacientes entre 25 y 35 años, sin 
enfermedades sistémicas, no bebedores y que no tomaban ningún medicamento, de los cuales 5 eran fumadores y 5 no fumadores. El criterio utilizado para definir a un paciente como fumador fue el dictado por National Health Interview Survey (NHIS), es decir, un adulto que ha fumado al menos 100 cigarrillos. Para aislar las células se utilizó el método de disociación enzimática, la capacidad proliferativa de las CMM se midió utilizando el ensayo de proliferación de bromuro y difeniltetrazolio (MTT). Para evaluar la diferenciación osteogénica se utilizaron las tinciones con rojo de alizarina, análisis de expresión génica de la fosfatasa alcalina y los genes de osteocalcina. Los resultados del ensayo de proliferación MTT mostró que la tasa de absorbancia media de las células madres de la pulpa dental en los no fumadores fue significativamente mayor que en el grupo de los no fumadores. A la tinción de rojo de alizarina, se observó menos depósitos de calcio luego de 1 días de inducción osteogénica en los pacientes fumadores. También se pudo observar que las expresiones de la fosfatasa alcalina y los genes de osteocalcina fueron significativamente mayores en las CMM de pulpa dental de los pacientes no fumadores. Las conclusiones que arroja este estudio son que la viabilidad celular del grupo de fumadores es reducida, por lo tanto, fumar si tiene un impacto negativo en la proliferación y el potencial regenerativo de las células madres mesenquimales de la pulpa dental ${ }^{2}$.

En otro estudio similar, los pacientes seleccionados fueron 3 no fumadores y 3 fumadores, cumpliendo el requisito de haber fumado durante los últimos 30 días, la indicación de extracción dentaria fue por motivos de ortodoncia. La proliferación de las células madre del ligamento periodontal (CMLP) se evaluó mediante MTT, cultivando 1.000 células por pocillos, en placas de 24 pocillos, esto durante 7 días. Las revisiones se realizaron los días: $0,1,3,5$ y 7 . Los resultados demostraron que la tasa de proliferación de las CMLP en fumadores disminuye en comparación a la de los no fumadores, en el día 3 la proliferación de los no fumadores es 1.79 veces mayor que en el grupo de fumadores, luego el día 5 la diferencia es de 2.53 y finalmente el día 7 es de 2.88. El análisis de migración celular, también lo realizaron mediantes cultivos y a las células sembradas le hicieron heridas, posterior a esto se siguió protocolo de lavado para finalmente tomar fotomicrografías a las $0,4,8,12$ y 24 horas, para obtener resultados midieron la reducción promedio de la zona en los diferentes momentos en que se tomaron las fotomicrografías comparándolas con el momento 0. Obteniendo como resultados, que la reducción de la herida fue menor en pacientes fumadores, teniendo diferencias significativas a las 12 y 24 horas. Esto demuestra que fumar, reduce la capacidad de migración de las CMLP [37].

Otro estudio clínico muestra el efecto biológico negativo sobre las CMM orales, aquí se aislaron cuatro tipos de células madres mesenquimales del periodonto humano: células madre del ligamento gingival (CMLG), células madre del ligamento periodontal (CMLP), células madre del tejido gingival (CMTG) y células madre del hueso alveolar (CMHA). Todas las muestras fueron extraídas de tejido periodontal de terceros molares impactados con indicación de extracción por ortodoncia, la población analizada fueron 18 mujeres entre 18 a 22 años y que no presentaran enfermedades sistémicas ni complicaciones dentales asociadas a las piezas a extraer. La solución de nicotina $(20 \mathrm{mM})$ se diluyó en agua libre de endotoxinas para así poder obtener las concentraciones que serían utilizadas en los tratamientos celulares, las cuales se clasificaron en alta, intermedia y bajas dosis. Las células fueron sembradas en placas y se incubaron durante $24 \mathrm{~h}$, para luego ser tratada con la solución de nicotina durante 24 y $48 \mathrm{~h}$. Para evaluar el efecto de citotoxicidad de la nicotina pasada las 24 y $48 \mathrm{~h}$, se utilizó el ensayo MTT, además de exámenes histológicos y la lectura de la densidad óptica con lector de microplacas. Se obtuvo como resultado que las altas concentraciones de nicotina indujeron muerte celular en los cuatro tipos de células aisladas, en cambio las dosis intermedias de nicotina indujeron autofagia en todas las células, además de disminuir significativamente el número de las CMHA después de 24 h, en cambio, las CMLG y las CMTG se vieron levemente afectadas, pero luego de 48 horas. Las dosis bajas de nicotina no mostraron cambios significativos en relación a los controles. Como conclusión de este estudio se puede mencionar que la citotoxicidad de la nicotina fue dosis y tiempo dependiente, también su reacción fue distinta dependiendo del origen de las células madres, sin embargo, queda en claro que la nicotina tiene efectos negativos sobre ellas ${ }^{36}$.

En un estudio a partir de premolares, las CMLP obtenidas a través de raspado de $1 / 3$ del tejido del ligamento periodontal, fueron cultivadas in vitro con exposición a diferentes concentraciones de nicotina. Se utilizaron distintos métodos para evaluar las diferentes cualidades de las CMM y como la nicotina puede influir en ellas. Se utilizó citómetro de flujo para detectar la capacidad de proliferación de las CMLP expuestas a concentraciones variadas de nicotina. También se usó tinción con rojo de alizarina para observar la formación de nódulos mineralizados después de que las CMLP fueron 
estimuladas con nicotina, finalmente se utilizó la reacción en cadena de la polimerasa en tiempo real (RT-PCR) y transferencia de Western para detectar los cambios en el potencial osteogénico. Los resultados fueron concluyentes, cuando la concentración de nicotina era de $10^{-4} \mathrm{~mol} \cdot \mathrm{L}$ la proliferación de CMLP podía suprimirse en pacientes fumadores después de 3 días, de igual forma la cantidad de nódulos mineralizados se redujo posterior a la diferenciación osteogénica. Al RT-PCR y Western Blot se observó que niveles de expresión de la fosfatasa alcalina y osteocalcina fueron más bajos en el grupo de fumadores. Es decir, la nicotina suprime la proliferación y la capacidad ostegénica de las CMLP ${ }^{38}$. De igual manera, en otro estudio con 10 pacientes, 5 fumadores y 5 no fumadores, sometidos a extracción dentaria por motivos de ortodoncia, las CMLP fueron aisladas y cultivadas para realizar los estudios de PCR en tiempo real para microARN-18b, mediante el cual pudieron seguir los procesos de proliferación, migración y diferenciación, finalmente llegaron a la conclusión de que la nicotina tiene un efecto inhibidor sobre todo los procesos mencionados y que el microARN-18b puede ser un regulador en los cambios funcionales asociados a la nicotina ${ }^{39}$.

\section{Conclusión}

Los componentes del tabaco son perjudiciales para las características biológicas de las células madres orales, especialmente para las capacidades de regeneración.

Todos los autores declaran que no existen conflictos potenciales de interés con respecto a la autoría y / o publicación de este artículo. All authors declare no potential conflicts of interest with respect to the authorship and/or publication of this article.

\section{Referencias}

1. Madrigal M, Rao KS, Riordan NH. A review of therapeutic effects of mesenchymal stem cell secretions and induction of secretory modification by different culture methods. J Transl Med.2014;12(1):1-14.

2. Kamel AHM, Kamal SM, Abubakr N. Effect of smoking on the proliferation capacity and osteogenic potential of human dental pulp stem cells (DPSCs).Dent Med Probl. 2020;57(1):1-6.

3. De Becker A, Riet IV. Homing and migration of mesenchymal stromal cells: How to improve the efficacy of cell therapy? World J Stem Cells.2016;8(3):73-87.

4. Taketani T, Oyama C, Mihara A, et al. Ex vivo expanded allogeneic mesenchymal stem cells with bone marrow transplantation improved osteogenesis in infants with severe hypophosphatasia.Cell Transplant. 2015;24(10):1931-1943.

5. Huang S, Xu L, Zhang Y, et al. Systemic and local administration of allogeneic bone marrow-derived mesenchymal stem cells promotes fracture healing in rats. Cell Transplant. 2015;24(12):2643-2655.

6. Murena L, Canton G, Vulcano E, et al. Treatment of humeral shaft aseptic nonunions in elderly patients with opposite structural allograft, BMP-7, and mesenchymal stem cells. Orthopedics. 2014;37:e201-206.

7. Ambrosio C, Zomer H, Vidane A, Gonçalves N. Mesenchymal and induced pluripotent stem cells: general insights and clinical perspectives. Stem Cells Cloning, 2015;8(1):125-134.

8. Liu J, Yu F, Sun Y, et al. Characteristics and Potential Applications of Human Dental Tissue-Derived Mesenchymal Stem Cells. Stem Cells. 2015;33(3):627-638.

9. Mori G, Ballini A, Carbone C, et al. Osteogenic Differentiation of Dental Follicle Stem Cells. Int J Med Sci. 2012; 9(6):480-487.

10. Assadollahi V, Mohammadi E, Fathi F, et al. Effects of cigarette smoke condensate on proliferation and pluripotency gene expression in mouse embryonic stem cells. J Cell Biochem. 2018;120(3):4071-4080.

11. Silva Vargas D. Efectos del condensado de humo de cigarrillo y nicotina sobre la migración y diferenciación miofibroblástica en fibroblastos gingivales humanos [Memoria para optar al título profesional de Bioquímico]. Facultad de Ciencias Químicas y Farmacéuticas, Universidad de Chile, Departamento de Biología y Bioquímica Molecular; 2010.

12. Behera SN, Xian H, Balasubramanian R. Human health risk associated with exposure to toxic elements in mainstream and sidestream cigarette smoke. Sci Total Environ. 2014;472:947-956.

13. Souto G. R., Queiroz-Junior C. M., Costa F. O., Mesquita R. A. Smoking effect on chemokines of the human chronic periodontitis. Immunobiology.2014;219(8):633-636.

14. Albuquerque EX, Pereira EF, Alkondon M, et al. Mammalian nicotinic acetylcholine receptors: From structure to function. Physiol Rev.2009;89(1):73-120. 
15. Schraufstatter IU, DiScipio RG, Khaldoyanidi SK. Alpha 7 subunit of nAChR regulates migration of human mesenchymal stem cells. J Stem Cells. 2009;4(4):203-215.

16. Hoogduijn MJ, Cheng A, Genever PG. Functional nicotinic and muscarinic receptors on mesenchymal stem cells. Stem Cells Dev. 2009;18(1):103-112.

17. Michailovici I, Harrington HA, Azogui HH, et al. Nuclear to cytoplasmic shuttling of ERK promotes differentiation of muscle stem/ progenitor cells. Development.2014;141(13): 2611-2620.

18. Zhu W., Liang M. Periodontal Ligament Stem Cells: Current Status, Concerns, and Future Prospects. Stem Cells Int. 2015; 2015:1-11.

19. Greenberg J.M., Carballosa C.M., Cheung H.S. Concise Review: The Deleterious Effects of Cigarette Smoking and Nicotine Usage and Mesenchymal Stem Cell Function and Implications for Cell-Based Therapies. ST CELLS Transla Med.2017;6(9):1815-1821.

20. Wahl EA, Schenck TL, Machens HG, et al. Acute stimulation of mesenchymal stem cells with cigarette smoke extract affects their migration, differentiation, and paracrine potential. Sci Rep. 2016;6(1):1-9.

21. Zeng HL, Qin YL, Chen HZ, et al. Effects of nicotine on proliferation and survival in human umbilical cord mesenchymal stem. J Biochem Mol. 2014;28(4):181-189.

22. Matson JP, Cook JG. Cell cycle proliferation decisions: The impact of single cell analyses. FEBS J. 2016;284(3):362375.

23. Ayo-Yusuf OA, Olutola BG. Epidemiological association between osteoporosis and combined smoking and use of snuff among South African women. Niger J Clin Pract. 2014;17(2):174-177.

24. Pearson RG, Clement RG, Edwards KL, et al. Do smokers have greater risk of delayed and non-union after fracture, osteotomy and arthrodesis? A systematic review with metaanalysis. BMJ Open. 2016;6(11):1-10.

25. Scolaro JA, Schenker ML, Yannascoli S et al. Cigarette smoking increases complications following fracture: A systematic review. J Bone Joint Surg Am. 2014;96(8):674-681.

26. Sopori M. Effects of cigarette smoke on the immune system. Nature Reviews Immunology. 2002;2(5):372-377.

27. Benowitz NL, Hukkanen J, Jacob P. Nicotine chemistry, metabolism, kinetics and biomarkers. Handb Exp Pharmacol.2009;(192):29-60.

28. Lindell G, Lunell E, Graffner H. Transdermally administered nicotine accumulates in gastric juice. Eur J Clin Pharmacol.1996;51(3-4):315-318.

29. Wu L, Yang K, Gui Y, Wang X. Nicotine-upregulated miR-30a arrests cell cycle in G1 phase by directly targeting CCNE2 in human periodontal ligament cells. Biochem Cell Biol. 2019;0(1):2-29.

30. Du A, Cheng Y, Zhao S, et al. MicroRNA expression profiling of nicotine-treated human periodontal ligament cells. J Oral Sci. 2019;61(2):206-212.

31. Yu W, Hu B, Shi X, et al. Nicotine inhibits osteogenic differentiation of human periodontal ligament cells under cyclic tensile stress through canonical Wnt pathway and $\alpha 7$ nicotinic acetylcholine receptor. J Periodont Res. 2018; 53(4):555564.

32. Chen CS, Lee SS, Yu HC, et al. Effects of nicotine on cell growth, migration, and production of inflammatory cytokines and reactive oxygen species by cementoblasts. J Dent Sci. 2015;10(2):154-160.

33. Kallala R, Barrow J, Graham SM, et al. The in vitro and in vivo effects of nicotine on bone, bone cells and fracture repair. Expert Opin Drug Saf. 2015;12(2):209-233.

34. Hermizi H, Faizah O, Lima-Nirwana S, et al. Nicotine impaired bone histomorphometric parameters and bone remodeling biomarkers in Sprague Dawley male rats. Ann Microsc. 2007; 7:10-24.

35. Fang Y, Svoboda KH. Nicotine inhibits myofibroblast differentiation in human gingival fibroblasts. J Cell Biochem. 2005;95(6):1108-1119.

36. Moga M, Bosca AB, Soritau O, et al. Nicotine Cytotoxicity on the Mesenchymal Stem Cells Derived from Human Periodontium. Romanian Biotech Lett.2016;21(4):11763 - 11772.

37. Ng TK, Huang L, Cao D, et al. Cigarette smoking hinders human periodontal ligament-derived stem cell proliferation, migration and differentiation potentials. Sci Rep. 2015;5(1):1-7.

38. Luan Y, Deqin Y. The effect of Toll-like receptor 4 in nicotine suppressing the osteogenic potential of periodontal ligament stem cells.West China J of Stomatology.2017; 35(4):368-372.

39. Baodi H, Jiandong D, Yingjie Z. MicroRNA-18b mediates the inhibitory effects of nicotine on periodontal ligamentderived stem cell. Int J Clin Exp Med.2018; 11(4):3604-3611.

Publisher's Note: This article is an open access article distributed under the terms and conditions of the Creative Commons Attribution(CC BY) license (http://creativecommons.org/licenses/by/4.0/)

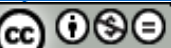

